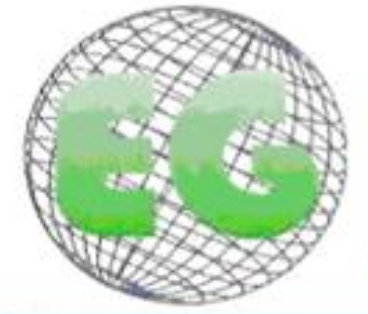

No 39

\title{
Enunciados sobre la atención domiciliaria en el panorama mundial: revisión narrativa
}

Enunciados sobre a atenção domiciliar no cenário mundial: revisão narrativa

Statements about home care in world stage: narrative review

\section{*Oliveira, Stefanie Griebeler ${ }^{* *}$ Kruse, Maria Henriqueta Luce ${ }^{* * *}$ Sartor, Silvia Francine ${ }^{* * * *}$ Echevarría-Guanilo, Maria Elena}

\begin{abstract}
*Doctoranda en Enfermería, Universidad Federal de Rio Grande do Sul (UFRGS). Profesora Asistente I de la Facultad de Enfermería de la Universidad Federal de Pelotas (UFPEL). E-mail: stefaniegriebeleroliveira@gmail.com ${ }^{* *}$ Enfermera. Doctorado en Educación. Profesor Asociado IV de la Escuela de Enfermería, UFRGS ${ }^{* * *}$ Estudiante de Enfermería, UFPEL. Estudiante en Movilidad Académica. Indiana University, Bloomington. ${ }^{* * * *}$ Doctora en Enfermería. Profesor Adjunto de la Facultad de Enfermería, UFSC. Brasil.
\end{abstract}

\section{Palabras clave: Servicios de Atención de Salud a Domicilio; Personas Imposibilitadas; Cuidadores Palavras chave: Serviços de Assistência Domiciliar; pacientes domiciliares; cuidadores} Keywords: home care services; homebound persons; caregivers

\section{RESUMEN}

Se objetivó conocer las definiciones e implicaciones de la atención domiciliaria abordadas en artículos científicos. Se trata de una investigación bibliográfica, revisión narrativa. La búsqueda fue realizada en la base de datos National Library of Medicine National Institutes of Health (PUBMED), en diciembre de 2012. Los descriptores Mesh Database utilizados fueron: home care services; patient care team; homebound persons y caregiver. Fueron encontrados 2244 publicaciones. El proceso de inclusión y exclusión se dividió en dos etapas: la primera fue la lectura de los títulos y resúmenes para verificación del tema abordado "atención domiciliaria", siendo seleccionados 253 títulos y resúmenes. La segunda fue el llenado del cuadro de informaciones elaborado en el programa Microsoft Excel 2007, siendo concluida con 209 resúmenes. Para el análisis en la integra, 72 artículos fueron seleccionados para análisis de contenido e identificación de los enunciados. Fueron elaboradas tres categorías enunciativas: Atención domiciliaria: ¿Qué es? ¿Para qué sirve?; Atención Domiciliaria: Fragilidades y potencialidades; Cuidador Familiar: fatiga y aislamiento.

\section{RESUMO}

Objetivou-se conhecer as definições e implicações da atenção domiciliar abordadas em artigos científicos. Trata-se de uma pesquisa bibliográfica, do tipo revisão narrativa. A busca foi realizada na 
base de dados National Library of Medicine National Institutes of Health (PUBMED), em dezembro de 2012. Os descritores Mesh Database utilizados foram: home care services; patient care team; homebound persons; caregiver. Foram encontrados 2244 títulos de publicações. O processo de inclusão e exclusão dividiu-se em duas etapas: a primeira foi a leitura dos títulos e resumos para verificação do tema abordado "atenção domiciliar", sendo selecionados 253 títulos e resumos. A segunda, foi o preenchimento do quadro de informações elaborado no programa Microsoft Excel 2007, sendo concluída com 209 resumos. Para análise na integra, 72 artigos foram selecionados para analise de conteúdo e identificação dos enunciados. Foram elaboradas três categorias enunciativas: Atenção domiciliar: o que é? Para que serve?; Atenção domiciliar: Fragilidades e potencia; Cuidador familiar: fadiga e isolamento.

\section{ABSTRACT}

It was aimed to know the definitions and implications of home care approached in scientific articles. It is a bibliographic research, a narrative review. The search was performed in the database National Library of Medicine National Institutes of Health (PUBMED), in December of 2012. The descriptors Mesh Database used were: care services; patient care team; homebound persons and caregiver. It was found 2244 titles of publications. The process of inclusion and exclusion divided itself in two steps: the reading of the titles and abstracts to verify the approached theme, "home care", being selected 253 titles and abstracts. The second one was the filling of the frame of information, performed in the Microsoft Excel 2007 program, being concluded with 209 abstracts. In the analysis in a full form, 72 articles were selected to analysis of content and identification of statements. It was performed three enunciative categories: home care: what is it? For what is it used?; Home care: Fragilities and Potentialities; Familiar Caregiver: fatigue and isolation.

\section{INTRODUCCIÓN}

La atención domiciliaria (AD) es presentada como una de las maneras de atender las demandas que emergen de los cambios en el proceso salud-molestia. La Organización Mundial de la Salud (OMS), en el inicio del siglo XXI, justificó la asistencia domiciliaria como una necesidad frente a los cuidados a largo plazo en las molestias crónicas, que vienen aumentando con la transición demográfica y epidemiológica vivida en los últimos años ${ }^{(1)}$. Históricamente, el cuidado en el domicilio surgió entre las enfermeras norteamericanas, al final del siglo XIX a través de la Visiting Nursing Association ${ }^{(2)}$. Fue, originalmente, llamado de home care, significando lar y cuidado, respectivamente, siendo en el idioma portugués traducido como cuidados realizados en el domicilio.

En Brasil, la política pública más actual, propuesta por el Ministerio de Salud (MS) por medio de la orden $n^{\circ} 963$ de 27 de mayo de 2013, fue el "Programa Melhor em Casa" (Programa Mejor en Casa), que establece diferentes modalidades de atención, dependiendo de la caracterización del paciente, del tipo de atención y de los procedimientos utilizados para la realización del cuidado ${ }^{(3)}$.

Por haber posibles diferencias entre los distintos países, se ha creído ser importante el resalte de estas informaciones y, además, hacer el análisis de frente al encontrado. Por cambiar la región, las costumbres, las creencias, y los pensamientos, es necesario repensar la importancia que todo eso tiene y su impacto en la salud, y principalmente en el cuidado cerca del enfermo y su familia. Al tener conocimiento de esto, se esperó encontrar las formas de organización y comprensión cerca de la $A D$ en los distintos países, como África del Sur, Estados Unidos, Brasil, Taiwán y Canadá, entre otros abordados al discurrir del texto. Incluso conteniendo muchas diferencias entre las distintas nacionalidades, muchos de ellos poseen detalles parecidos y, a través de ello, se puede hacer el análisis de contenido, principalmente por el hecho de haber muchas publicaciones, las cuales permiten un grande acúmulo de datos e informaciones. 
Ante estas propuestas, apoyadas en discursos que organizan y producen esa modalidad de cuidados, se objetivó conocer, en los artículos científicos que tratan del asunto, cómo se constituyó esta modalidad de atención a la salud. De esa forma, se propuso como pregunta que norteó la revisión narrativa: ¿Qué enunciados acerca de la atención domiciliaria son encontrados en la literatura científica que aborda el tema? ¿Cómo ellos se presentan en relación al escenario mundial?

\section{MATERIALES Y MÉTODOS}

Se trata de una inmvestigación bibliográfica, del tipo revisión narrativa, que permite describir el "estado del arte" de un determinado asunto, desde un punto de vista teórico o contextual. En esta modalidad de revisión, no hay necesidad de informar las fuentes utilizadas, ni criterios de inclusión para selección de los trabajos ${ }^{(4)}$. Sin embargo, las etapas que establecieron la inclusión de las publicaciones de la base de datos seleccionada en la presente revisión fueron descritas, de forma que permitan evidenciar la organización y sistematización de la búsqueda, la inclusión o exclusión de trabajos, posibilitando su reproducción.

La búsqueda fue realizada en la base de datos National Library of Medicine National Institutes of Health (PUBMED), en diciembre de 2012, sin demarcación temporal, proporcionando que encontrásemos el primer artículo en 1991, abordando este tema. Los descriptores Mesh Database utilizados fueron con la siguiente intersección: ((("home care services"[MeSH Terms]) AND "patient care team"[MeSH Terms]) AND "homebound persons"[MeSH Terms]) OR "caregivers"[MeSH Terms]. Con la intención de favorecer la delimitación (especificidad) de la búsqueda, entre las distintas herramientas de búsqueda disponibles en esta base de datos, fueron utilizados como límites de estrategia los siguientes filtros: Abstract available, Humans, Clinical Trial, Clinical Trial, Phase I, Randomized Controlled Trial, Clinical Trial, Phase II, Clinical Trial, Phase III, Clinical Trial, Phase IV, Evaluation Studies, Comparative Study, Controlled Clinical Trial, Journal Article, Multicenter Study, Validation Studies, English, Portuguese, Spanish, AIDS, Cancer y History of Medicine. Como resultado de la estrategia de búsqueda, fueron encontrados 2244 títulos de publicaciones. A partir de estos resultados, el proceso de inclusión y exclusión se dividió en dos etapas: Primera etapa - lectura de títulos y resúmenes para verificación del tema abordado "atención domiciliaria", siendo seleccionados 253 títulos y resúmenes. La segunda etapa - inclusión de informaciones en el cuadro de datos elaborado en el programa Microsoft Excel 2007 contemplando las informaciones: título, autor, año, país, institución responsable de la investigación, resumen, área a la que estaba destinado, los sujetos de investigación, el abordaje de la investigación, el tipo de estudio utilizado, la perspectiva teórica y la revista de publicación. Fueron excluidos los relatos de experiencia, reflexiones y revisiones, totalizando 209 artículos analizados.

Para los pasos subsecuentes fueron considerados criterios de inclusión de los artículos para análisis en la integra: referir/mencionar las perspectivas de los involucrados en la atención domiciliaria y tener el artículo disponible online. Fueron seleccionados 72 artículos ${ }^{(5-76)}$ sometidos a análisis cualitativo de contenido ${ }^{(77)}$. En el análisis, fueron identificados los enunciados que constaban en estas publicaciones. Esos enunciados tienen el poder de producir los contenidos tratados, y las revistas científicas son entendidas como el lugar privilegiado para la circulación de esos discursos ${ }^{(78)}$. 


\section{RESULTADOS Y DISCUSIÓN}

Después del análisis, fueron establecidas tres categorías de enunciados relacionados a la atención domiciliaria y están relacionados a lo que sería esta modalidad asistencial, potencialidades y fragilidades, la fatiga y aislamiento vivido por el cuidador domiciliario. Atención domiciliaria: ¿Qué es? ¿Para qué sirve?; Atención Domiciliaria: Fragilidades y potencialidades; Cuidador Familiar: fatiga y asilamiento.

\section{Atención Domiciliaria: ¿Qué es? ¿Para qué sirve?}

En esta categoría, apuntamos enunciados que explican la atención domiciliaria, apuntan sus objetivos y determinan para quien se direcciona esta modalidad de cuidado. La mayor parte de los estudios sobre la atención domiciliaria están relacionados con la oferta de cuidados paliativos ${ }^{(5-23)}$, de este modo, parece posible afirmar que el domicilio es un lugar para estar el enfermo cuando no hay posibilidades de cura, ya que en los artículos analizados se observó que cuidadores, familiares y pacientes, recurrentemente refieren la muerte y la terminalidad.

En estudios de Australia ${ }^{(8-11)}$, se identifican convergencias y divergencias en relación al enfrentamiento de la muerte en el domicilio. Los prestadores de cuidados paliativos en la atención domiciliaria apuntan esta modalidad asistencial como un servicio utilizado por personas con una enfermedad terminal, con la cual, si no hubiera "aceptación de la muerte", los pacientes pueden quedarse alarmados por relacionarlos con el fin de la vida. La mayoría de los cuidadores refiere que aunque los cuidados paliativos no preparen para la muerte, promueven bienestar, lo que podría facilitar y humanizar el proceso de morir ${ }^{(8)}$. Familiares consideran el acompañamiento del proceso de muerte y morir en el domicilio "maravilloso" o "adorable" (9), que posibilita la despedida ${ }^{(10)}$ y sensación de satisfacción de las necesidades de los moribundos ${ }^{(9,11)}$.

En una investigación en Israel, se apuntó la preferencia por cuidados de fin de vida en el domicilio, pues la relación con el paciente sería más próxima ${ }^{(12)}$, factor que también justifica la elección de padres italianos ${ }^{(13)}$, americanos ${ }^{(14)}$ y australianos ${ }^{(15)}$ de poder cuidar de sus hijos en casa en el final de la vida. La manutención de "vida normal" también influyó en los padres ingleses a optar por el cuidado domiciliario de sus hijos con enfermedad terminal ${ }^{(17)}$. La preferencia por los cuidados paliativos en el domicilio también está relacionada a la posibilidad de acompañar al niño en relación a la nutrición, alivio de síntomas y bienestar ${ }^{(13,15,17)}$.

Además, los cuidados domiciliarios significan proximidad con el paciente en situación de terminalidad, por mantenerlo dentro de la dinámica familiar e vida social ${ }^{(5)}$. Los pacientes suecos relatan los cuidados domiciliarios, junto a sus familiares, como un privilegio, destacando el poder tener acceso a sus pertenencias, preservando su independencia. También los cuidados y las informaciones recibidas por el personal de salud fueron adecuados y proporcionaron seguridad ${ }^{(7)}$.

En estudios realizados en Suecia ${ }^{(5)}$ y en Dinamarca ${ }^{(18)}$, los pacientes refirieron sentirse seguros y confortables en sus casas, por facilitar el cuidado paliativo para los pacientes y sus familiares. Sin embargo, por momentos los pacientes pasan a sentirse frustrados y/o culpados por sentirse una carga para el familiar más próximo, así como para la sociedad ${ }^{(5)}$. 
En Australia, la seguridad en la atención domiciliaria parece estar relacionada a la confianza en el sistema de salud. Los padres refirieron confiar en el sistema de salud, principalmente al percibir sinceridad en el cuidado de los profesionales y sentirse confortables para hacer preguntas y obtener informaciones sobre el tratamiento del niño ${ }^{(15)}$. A pesar de acreditar que el paciente está más seguro recibiendo cuidados domiciliarios, y que la atención es de calidad y eficiente, en Canadá, los cuidadores sienten menos seguridad en cuidar del paciente en este ambiente ${ }^{(19)}$.

La atención domiciliaria es percibida como un cuidado propiciado en un ambiente favorable, próximo de la familia y del ambiente familiar. En estudio Sueco, se identificó que los familiares conceden a la "atmósfera" del domicilio un efecto curativo sobre el paciente ${ }^{(21)}$, y que estos cuidados promueven más tiempo con la familia y amigos ${ }^{(22)}$, además de permitir cumplir los deseos del familiar enfermo ${ }^{(23)}$. En Brasil, la atención domiciliaria también fue relacionada a la importancia de la familia como base de apoyo afectivo y social para los pacientes ${ }^{(24)}$. En Irlanda del Norte, muchos cuidadores relatan no tener elección, y aceptan la atención domiciliaria para cumplir los deseos de la persona enferma de morir en casa ${ }^{(20)}$.

En relación a si el ambiente más adecuado para el cuidado en el final de la vida sería el domicilio o el hospital, se identificaron divergencias. En Turquía, los cuidadores consideraran la casa como el ambiente más adecuado ${ }^{(6)}$. En estudio de Lesoto ${ }^{(25)}$, los cuidadores refirieron mejores resultados cuando son hospitalizados, ya que el gobierno ofrece el tratamiento. Aún mejores resultados pueden relacionarse con el manejo de carencia alimentaria, especialmente entre los portadores de $\mathrm{VIH}$, necesitando optimismo y organización de los cuidadores.

La atención domiciliaria también es percibida por los familiares como deshospitalización. Los familiares se sienten frustrados cuando el paciente recibe el alta del hospital antes de estar curado, ya que relacionan el alta a la cura, revelando que no aceptan salir del hospital en cuanto que los pacientes son incapaces de cuidar de sí mismos. En este sentido, los cuidados domiciliarios son vistos como un medio de disminuir la carga de trabajo de los hospitales, destacando la importancia de la preparación previa de los cuidadores domiciliarios, para que puedan conferir cuidado adecuado al paciente en casa ${ }^{(26)}$.

En algunas situaciones, escoger la atención domiciliaria no ocurre por parte de los familiares, pero si por atender al deseo del enfermo o por la imposibilidad del acceso a otras formas de cuidado ${ }^{(23)}$. Muchas familias sienten inseguridad en relación a la situación del paciente en el domicilio, especialmente frente a alteraciones en su salud y saber si deben o no encaminarlo al hospital. Entre tanto, cuando las condiciones del domicilio son adecuadas, las enfermeras tienden a incentivar a los familiares a mantener sus familiares enfermos en casa ${ }^{(16)}$.

Para la mayoría de las familias, el cuidado paliativo en casa facilita la "desprofesionalización" al proceso de morir, ya que es la familia la principal fuente de cuidados para el niño ${ }^{(17)}$. En Israel, más de $90 \%$ de los cuidadores del programa homecare refieren que la muerte en casa es una experiencia positiva, tanto para sí cuanto para sus seres queridos que murieron ${ }^{(12)}$.

El miedo a ser infectado por alguna enfermedad fue identificado en dos estudios. Entre italianos, portadores de $\mathrm{VIH}$, surgió el miedo de infectar a algún familiar. Miedo también presentado por los cuidadores. Sin embargo, destacase que en casa, estos 
recibirían más atención y cariño (27). En Botsuana, los cuidadores también manifestaron miedo a la infección, sin embargo, relataron que utilizar guantes podría herir la relación de amor con su ente querido ${ }^{(28)}$.

\section{Atención Domiciliaria: Fragilidades y Potencialidades}

Además de políticas públicas que organicen y apoyen la atención domiciliaria, depende de recursos económicos, materiales y humanos para que esta se materialice. Los artículos estudiados apuntan especialmente la falta de comunicación y de información como fragilidades encontradas en la realización de la asistencia domiciliaria. La falta de comunicación afecta tanto la parte económica de la familia como a las relaciones entre personal de salud-paciente-familia. En estudio realizado en Turquía, la falta de comunicación es apuntada como un prejuicio para el alta hospitalaria $^{(6)}$ y en los cuidados domiciliarios. En Malawi ${ }^{(29)}$ y en Kenia ${ }^{(30)}$, la falta de información por parte del personal de salud se presentó como limitación para la prestación de cuidados eficientes y de calidad en el domicilio. En Japón ${ }^{(31)}$ y en Irlanda del Norte(32) se refirió la falta de información del pronóstico del paciente como factor de inseguridad cara al futuro. En Dinamarca, se relató la falta de información por parte de los médicos acerca de las condiciones de salud de los pacientes ${ }^{(33)}$. En Inglaterra, las informaciones sobre beneficios financieros y fuentes de ayuda externa al servicio de salud no siempre ocurrirían ${ }^{(34)}$.

La estructura del domicilio para la realización de la atención domiciliaria también es tema abordado en los artículos analizados. La falta de saneamiento ${ }^{(26)}$ y carencia de alimentos nutritivos son apuntados como dificultades en el continente africano ${ }^{(26,35-37)}$. En la Suazilandia, país de África Austral, niños cuidadores describieron sentimientos de tristeza frente a la falta de oportunidad de escoger y a la necesidad de aceptar las condiciones, como falta de acceso, de alimentación adecuada, de apoyo físico y emocional, o informaciones sobre los servicios que podrían ampararlos. Esa realidad, niños cuidadores, se presenta en países con expresivo número de huérfanos debido a la mortalidad de adultos con SIDA, lo que reduce sustancialmente el número de adultos que pueden cuidar de los enfermos ${ }^{(38)}$

En Kenia, el espacio físico también fue apuntado como una cuestión fundamental. Muchas veces, la casa es pequeña para acomodar a una persona con SIDA y otros miembros de la familia. En este sentido, los cuidados en domicilio no representan solo costos sociales y económicos, sino también posibles riegos de infección, ya que los miembros de la familia muchas veces no conocen el diagnóstico y pueden no tomar las precauciones necesarias ${ }^{(30)}$.

Las dificultades estructurales para los cuidados domiciliarios extrapolan el ambiente de la casa, e incluyen el transporte disponible, lo cual determina las condiciones de acceso y accesibilidad. En Africa del Sur ${ }^{(26)}$ y en Inglaterra, autores destacan dificultades en las áreas rurales, principalmente por la distancia y la falta de transporte gratuito $^{(39)}$ para acceso a los servicios de salud. En Italia, la desigualdad al acceso a los servicios de salud coloca a pacientes y familias, socialmente desfavorecidos, en una desventaja adicional, en lo que se refiere a la salud ${ }^{(27)}$. Entre los brasileños, son apuntados como aspectos que dificultan el acceso: la falta de recursos económicos, tanto para la alimentación cuanto para la compra de medicamentos, las condiciones inadecuadas de vivienda y la deficiencia del transporte público. Tales dificultades van más allá del estigma, resultando en sufrimiento y tristeza. Paciente y cuidadores 
dependen de ambulancias y transporte públicos insuficientes y lentos en las ciudades brasileñas $^{(40)}$.

Cuando se observa la cuestión financiera, principalmente en Botsuana, país africano, uno de los desafíos más citados por las familias es la falta de recursos para cubrir los costos con el cuidado de sus familiares enfermos, ya que el gobierno contribuye solo con el $9 \%$ de esos gastos ${ }^{(41)}$. Muchas veces las familias pierden dinero con curanderos que prometen la cura del SIDA ${ }^{(28)}$. Aunque en los Estados Unidos el homecare sea de bajo costo, comparado al costo de la hospitalización, la atención domiciliaria origina gran impacto en la renta de la familia ${ }^{(42)}$.

El apoyo, tanto estructural (materiales y recursos humanos), como material (beneficios financieros) de los servicios de salud también fueron aspectos apuntados, por afectar al desarrollo de la atención domiciliaria. En estudios de Corea ${ }^{(43)}$, Suecia ${ }^{(5)}$ y Estados Unidos ${ }^{(44,45)}$, los cuidadores relataron encontrar dificultad en dar apoyo emocional a los pacientes. En estudio holandés, se observó haber mayor número de médicos especialistas en atención hospitalaria que domiciliaria ${ }^{(46)}$. La demora en la atención, en el periodo nocturno y finales de semana, también fue apuntada por los profesionales de salud, la baja frecuencia de visitas recibidas por los enfermos y la falta de privacidad por la presencia del personal de salud, tornaría la casa parecida a una institución hospitalaria ${ }^{(47)}$. En África del Sur, la falta de equipamientos disponibles también fue apuntada como un problema ${ }^{(48)}$.

En un estudio de Suazilandia ${ }^{(38)}$, fue apuntado que la falta de equipamientos, como sillas de ruedas, pañales desechables y guantes, más que dificultar la realización de los cuidados domiciliarios, agravarían el estrés del cuidador. La garantía de que cuidadores domiciliarios cuenten con esos equipamientos, sería responsabilidad del gobierno ${ }^{(26)}$, como ocurre en Malawi (África), donde el gobierno identifica estrategias de sustentabilidad para apoyo familiar ${ }^{(28)}$.

En Inglaterra, entre los cuidadores, se identificó preocupaciones en relación a la responsabilidad asumida con los pacientes ${ }^{(17)}$, ya que hay pocos médicos y enfermeras para realizar las visitas y los cuidados domiciliarios. Aunque se señala que los cuidados mejoraron en los últimos diez años, aún existen dificultades, especialmente en relación a la falta de apoyo financiero, técnico y de información sobre los recursos disponibles para pacientes atendidos en domicilio ${ }^{(34)}$. En Estados Unidos, la gestión del dolor es fuente de gran preocupación, principalmente por la dificultad en el manejo de equipos y la necesidad de preparación de los cuidadores que los utilizarían ${ }^{(49)}$, ya que la dificultad con el control del dolor se agrava debido a la falta de apoyo del personal de salud ${ }^{(50-52)}$. Hay falta de política para la atención a los cuidadores, que permita aliviar su sobrecarga y que no sea restringido a los pacientes $^{(53)}$.

En Italia, se observó que el nivel de escolaridad del cuidador, o su status socioeconómico, afectaría la habilidad y la capacidad para identificar y obtener servicios apropiados de salud ${ }^{(27)}$. Entre familias de Zimbabue, fueron apuntadas las dificultades enfrentadas por los niños huérfanos de padres portadores de VIH. Incluso cuando la madre estaba viva, los hijos eran dejados con los abuelos, para que estas pudiesen trabajar. Muchos de los entrevistados manifestaron no concordar con esta situación, sin embargo, esta se tornaba inevitable cuando otro miembro de la familia no estaba dispuesto a cuidar de los niños. Sin embargo, si los ancianos muriesen, los 
niños se quedarían desamparados, por falta de apoyo social y financiero en ese país ${ }^{(54)}$.

\section{Cuidado Familiar: cansancio y aislamiento}

Esta categoría reúne enunciados de artículos que apuntan características de los cuidados familiares, especialmente acerca de la sobrecarga física y emocional, provocada por la fatiga y el aislamiento social vividos por estas personas. Muchos investigadores, de distintos países, desarrollaron estudios para investigar la sobrecarga psicosocial y física que afecta los cuidadores ${ }^{(6,26,55-56)}$. En África del Sur, la sobrecarga entre los cuidadores estaría relacionada a la infección de $\mathrm{VIH}{ }^{(26,55)}$. Entre los participantes de un estudio de Canadá, la angustia emocional del cuidado fue relacionada a la restricción de actividades y a la pérdida de intimidad ${ }^{(56)}$. En otro estudio, los cuidadores manifestaron frustración cuando, para tener la oportunidad de descansar, necesitaban dejar al paciente más autónomo ${ }^{(57)}$.

La sobrecarga ${ }^{(58)}$ y la desvalorización ${ }^{(59)}$ fueron referidas por cuidadores de un estudio de Estados Unidos de América, pero, por otro lado, los preparó para momentos futuros $^{(58)}$. Tales momentos están llenos de inseguridad y necesidad de aprender a vivir con cambios en el comportamiento del paciente, lo que puede contribuir con el sufrimiento del cuidador ${ }^{(60)}$.

Un estudio en Hong Kong reveló que la mayoría de los cuidadores (83\%) manifiesta fatiga y estrés durante las 12 primeras semanas de cuidado. Sin ayuda o soporte, estos cuidadores pueden pasar a ser un segundo paciente en la familia ${ }^{(61)}$. Cuidadores de paciente con accidente cerebrovascular refirieron poco apoyo social en estudio de Taiwán. Fueron apuntados como causadores de estrés: falta de sueño y descanso inadecuado (76\%), mal humor (74\%), sentimiento de angustia y desamparo (72\%), ansiedad debida al estrés (72\%), sensación de incapacidad de manejar las situaciones con calidad $(72 \%)$, y sentimiento de fatiga $(70 \%){ }^{(60)}$. La falta de apoyo social estaría asociada a depresión, importante mediador en la sobrecarga, en la manifestación de ansiedad de los cuidadores y en las alteraciones de salud física ${ }^{(63)}$.

En Taiwán, se identificó la depresión como aspecto que agrava la salud de los cuidadores debido a altos niveles de estrés y ansiedad ${ }^{(64)}$, relacionados al escaso apoyo social que resulta en sobrecarga ${ }^{(65)}$. Esto sería inherente a las personas que conviven con el enfermo y su condición, especialmente a los hijos que participan emocionalmente de la situación-enfermedad ${ }^{(66)}$.

La imposibilidad de contar con un miembro de la familia para compartir la responsabilidad del cuidado también genera sobrecarga entre los cuidadores. La mayor parte de los cuidadores que participaron de un estudio en Australia relataron sentirse sobrecargados por no contar con alguien para compartir el cuidado del paciente terminal ${ }^{(67)}$, propiciando la privación del convivio cotidiano, social, ocupacional o familiar ${ }^{(68)}$. Situaciones como estas llevan a los cuidadores a sufrimiento emocional y físico ${ }^{(10)}$, una vez que pasan a asumir, además de la responsabilidad de su trabajo, el cuidado de un familiar, pudiendo favorecer la aparición de problemas de salud mental, como depresión y ansiedad ${ }^{(69)}$.

Las cuidadoras también tienden a manejar complejas demandas de cuidados por un largo tiempo y, al contrario de los hombres, optan por no pedir apoyo al personal de salud, a pesar de la fatiga. Interrupciones de actividades diarias aparentan tener mayor impacto sobre el cuidador ${ }^{(70)}$. En la nueva rutina de cuidados, surgen factores 
estresantes como tensión emocional, agotamiento físico debido al largo tiempo dedicado al cuidado del enfermo y por tener que desarrollar, también las tareas de casa, así como sentir pérdida de la independencia y crisis financiera ${ }^{(24,71)}$.

En Brasil, el foco del cuidador/familiar es el niño enfermo, cuidándolo en tiempo integral, y privándose de actividades de descanso. Esto lleva al cuidador a sentirse culpable por desear periodos de descanso, considerando tal pensamiento egoísta, pues el niño necesita de su atención y es su papel estar con él ${ }^{(72)}$. La falta de descanso también fue destacada entre cuidadores de un estudio de EUA, refiriendo la necesidad de levantarse durante la noche para cuidar del paciente ${ }^{(73)}$.

Estudios realizados en Suecia revelan que el familiar se siente presionado por la responsabilidad del cuidado sumado al sentimiento constante de preocupación, ya que permanece la mayor parte del tiempo con el paciente y necesita reconocer el momento de intervenir para ayudarlo ${ }^{(22)}$. Y ante esta situación, requiere enfrentar la sobrecarga, el aislamiento y las dificultades para recibir apoyo del equipo de salud ${ }^{(74)}$.

El aislamiento social entre los cuidadores también es bastante mencionado $(21,73,75)$. En estudio con cuidadores de Suecia, se identificó que ser un cuidador informal significaría quedarse preso en casa, comprometiendo las horas de descanso. Los cuidadores sienten doble responsabilidad: por el cuidado y por la culpa frente a alguna alteración del paciente, generando agotamiento. De esta forma, los cuidados paliativos en casa producen el aislamiento social del cuidador, lo que suena irónico una vez que este movimiento surgió para disminuir el aislamiento social del moribundo en el hospital. Aún, se relatan las pocas oportunidades que el cuidador tendría para descansar. A pesar de la posibilidad de solicitar ayuda hasta seis veces al día, estos prefieren no requerirla y no descansan debido a la frecuencia de cambios del personal de salud, y la solicitación de su permanencia por parte de estos. Se suma la inseguridad y el sentimiento de impotencia, por la dificultad de manejar cuestiones financieras al estar apartados del trabajo ${ }^{(21)}$.

Los estudios apuntan a que cuando se cuida de una persona de la familia, es necesario organizar una nueva rutina y redefinir las actividades diarias. La transición para un nuevo papel, de cuidador, o para la viudez implícita frente al fallecimiento del cónyuge, la duda sobre la disponibilidad del cuidado en casa y el mantenimiento de una buena relación con ese familiar, son cambios y adaptaciones frecuentes que el cuidador estará susceptible a enfrentar con el paso del tiempo ${ }^{(23)}$. En Ucrania, más allá de la fragilidad o falta de tiempo del cuidador, se observa la necesidad de saber cómo reaccionar frente al comportamiento agresivo del paciente, particularmente frente a la agresión verbal, que ocurre en la mayoría de los casos durante el curso de la demencia ${ }^{(76)}$.

Con todos estos desafíos, los cuidadores vienen cargados de frustración y dudas, principalmente por la complejidad y el riesgo de vida de enfermedades como el cáncer, junto con las consecuencias emocionales y psicológicas demandadas, cuidadosa coordinación de los distintos niveles de cuidados, o sea, primario, secundario, y terciario. Además, todas las personas envueltas en los cuidados paliativos en casa enfatizan que este tipo de cuidado exige trabajo adicional ${ }^{(5)}$. 


\section{CONSIDERACIONES FINALES}

El presente estudio posibilitó conocer los enunciados sobre la atención domiciliaria de artículos científicos localizados en la base de datos PUBMED y publicados entre 1991 y 2012. Tales discursos, elaborados por investigadores de distintos países, han constituido los profesionales de salud, produciendo rupturas y cambios sobre lo que piensan de las personas enfermas y sobre aquellos que los cuidan en el domicilio.

La lectura de los artículos permitió elaborar un panorama de esta modalidad asistencial, a partir de los enunciados de las revistas científicas, aquí destacadas como lugares privilegiados para la producción de discursos que podrían tener el poder de producir aquello de lo que se habla. Los enunciados producen la atención domiciliaria generalmente dirigida a pacientes en cuidados paliativos, considerando que muchos pacientes que necesitan de estos cuidados están fuera de posibilidad de cura. Al apuntar la atención domiciliaria como una modalidad dirigida a personas con enfermedades que amenazan la vida, destacan que tal modalidad contribuye para promover bienestar y satisfacción de las necesidades de los moribundos.

Los artículos describen que ser cuidador no es una elección voluntaria, y sí la atención a un deseo de la persona enferma. También adjudican discursos de cuidadores y pacientes que evalúan la atención domiciliaria como positiva, al considerar la proximidad del paciente con la familia, por permanecer en su ambiente familiar. Sin embargo, también destacan que muchos cuidadores se sienten sobrecargados, privados de necesidades básicas como sueño y buena alimentación, viviendo en aislamiento social por quedarse en torno del paciente y distante de sus actividades.

La lectura de los artículos permite evaluar facilidades y dificultades relacionadas a la atención domiciliaria, presentando situaciones particulares, como en el continente africano, donde el cuidado domiciliario representa un desafío, debido a muchos problemas, tales como la alta mortalidad de adultos por $\mathrm{VIH}$, dejando muchos niños huérfanos, hasta la falta de alimentos, debido a los bajos recursos financieros y la pobreza de muchas familias. El análisis también permite visualizar cómo cada país busca atender las demandas del proceso salud-enfermedad.

Los resultados también apuntan que los cuidados domiciliarios implican en la oportunidad de realizar cuidados en un ambiente favorable, permitiendo preservar las actividades familiares, sociales y el bienestar para el cuidado, además de proporcionar sentimiento de no abandono en situación de terminalidad. La falta de comunicación de los cuidadores con el personal de salud, en el momento del alta y durante la atención domiciliaria, también dificulta la realización de cuidados con calidad, de la misma forma que la infra-estructura domiciliaria, muchas veces precaria, dificultades económicas, alteraciones emocionales, sobrecarga de los cuidadores y dificultad de acceso en casos de interocurrencias o empeoramiento del estado de salud. Por otro lado, los textos refieren que acciones de capacitación de cuidadores contribuyen a la seguridad de los cuidadores.

Para concluir, las autoras sugieren que los aspectos identificados en la presente revisión puedan ser considerados en la programación de actividades desarrolladas en los programas de cuidados domiciliarios. 


\section{REFERENCIAS}

1. World Health Organization. Home-based long-term care: report of a WHO Study Group. Gêneva: World Health Organization 2000.

2. Dieckmann J. Home health administration: an overview. In: Harris MD. Handbook of home health care administration. $2^{\text {nd }}$ ed. Gaithersburg: Aspen 1997:3-14.

3. Brasil. Ministério Da Saúde. Portaria no 963, de 27 de maio de 2013: Redefine a Atenção Domiciliar no âmbito do Sistema Único de Saúde (SUS). Brasília: Ministério da Saúde 2013.

4. Rother ET. Revisão sistemática X revisão narrativa. Acta Paul Enferm 2007; 20(2): $1-2$.

5. Appelin G, Brobäck G, Bertero C. A comprehensive picture of palliative care at home from the people involved. Eur J Oncol Nurs 2005; 9(4): 315-24.

6. Alptekin S, Gönüllü G, Yücel I, Yaris F. Characteristics and quality of life analysis of caregivers of cancer patients. Med Oncol 2010;27(3): 607-17.

7. Melin-Johansonn C, Dling GO, Axelsson B, Danielson A. The meaning of quality of life: Narrations by patients with incurable cancer in palliative home care. Palliat Support Care 2008; 6(3): 231-8.

8. Sekelja N, Butow PN, Tattersall MH. Bereaved cancer carers' experience of and preference for palliative care. Support Care Cancer 2010; 18(9): 1219-28.

9. Mcgrath $P$, Joske D. Palliative care and haematological malignancy: a case study. Aust Health Rev 2002; 25(3): 60-6.

10. Wong $\mathrm{T}$, Ussher $\mathrm{J}$. Bereaved informal cancer carers making sense of their palliative care experiences at home. Health Soc Care Community 2009; 3(17): 274-82.

11. Burns C.M, Abernethy AP, Leblanc TW, Currow DC. What is the role of friends when contributing care at the end of life? Findings from an Australian population study. Psycho-oncology 2011; 20(2): 203-12.

12. Singer Y, Bachner YG, Shvartzman P, Carmel S. Home Death. The Caregivers' Experiences. Journal of Pain and Symptom Management 2005; 30(1): 70-4.

13. Beretta S, Polastri D, Clerici CA, Casanova M, Cefalo G, Ferrari A et al. End of Life in Children with Cancer: Experience at the Pediatric Oncology. Pediatr Blood Cancer 2010; 54(1): 88- 91.

14. Dussel V, Kreicbergs U, Hilden JM, Watterson J, Moore C, Turner BG et al. Looking Beyond Where Children Die: Determinants and Effects of Planning a Child's Location of Death. J Pain Symptom Manage 2009; 37(1): 33-43.

15. Monterosso L, Kristjanson LJ, Philips MB. The supportive and palliative care needs of Australian families of children who die from cancer. J Palliat Med 2009; 23(6): 52636.

16. Hirano Y, Yamamoto-Mitani N, Ueno M. Takemori S, Kashiwagi M, Sato I et al. Home Care Nurses' Provision of Support to Families of the Elderly at the End of Life. Qualitative Health Res 2011; 21(2): 199-213.

17. Vickers JL, Carlisle C. Choices and control: Parental Experiences in Pediatric Terminal Home Care. J Pediatr Oncol Nurs 2000; 17(1): 12-21.

18. Goldschmidt D, Schmidt L, Krasnik A, Christensen U, Groenvold M. Expectations to and evaluation of a palliative home-care team as seen by patients and carers. Support Care Cancer 2006; 14(12): 1232-40.

19. Stajduhar KI, Allan DE, Cohen SR, Heyland DK. Short Report: Preferences for location of death of seriously ill hospitalized patients: perspectives from Canadian patients and their family caregivers. Palliat Med 2008; 22(1): 85-8.

20. McLaughlin D, Sullivan K, Hasson F. Hospice at Home service: the carer's perspective. Support Care Cancer 2007; 15(2): 163-70. 
21. Brobäck G, Berterö $C$. How next of kin experience palliative care of relatives at home. Eur. J. Cancer Care 2003; 12(4): 339-46.

22. Milberg A, Strang $P$, Carlsson M, Börjesson S. Advanced Palliative Home Care: Next-of-Kin's Perspective. J Palliat Med 2003; 6(5): 749-56.

23. Wennman-Larsen A, Tishelman C. Advanced home care for cancer patients at the end of life: a qualitative study of hopes and expectations of family caregivers. Nordic College of Caring Sciences, Scand J Caring Sci 2002; 16(3): 240-7.

24. Gir E, Reis RK. Alterações no estilo de vida, necessidades e dificuldades enfrentadas pelos cuidadores de portadores de AIDS, no âmbito do domicílio. Rev Esc Enferm USP 2001; 4(35): 328-35.

25. Makoae MG, Jubber K. Confidentiality or continuity? Family caregivers' experiences with care for HIV/AIDS patients in home-based care in Lesotho. SAHARA J 2008; 5(1): 36-46.

26. Tshililo AR, Davhana-Maselesele M. Family experiences of home caring for patients with HIV/AIDs in rural Limpopo Province, South Africa. Nurs health Sci 2009; 11(2): 135-43.

27. Beccaro M, Costantini M, Merlo DF. Inequity in the provision of and access to palliative care for cancer patients. Results from the Italian survey of the dying of cancer (ISDOC). BMC Public Health 2007; 7(66): 1-13.

28. Lindsey $E$, Hirschfeld M, Tlou S, Ncube E. Home-based care in Botswana: experiences of older women and young girls. Health Care for Women International 2003; 24(6): 486-501.

29. Zimba EW, Mclnerney PA. The know ledge and practices of primary care givers regarding ho $\mathrm{m}$ e -base $\mathrm{d}$ care of HIV/AIDS children in Blantyre (Malawi). Curationis 2001; 24(3): 83-91.

30. Olenja JM. Assessing community attitude towards Home-based care for people with Aids (pwas) in Kenya. J Commun Health 1999; 24(3): 187-199.

31. Fukui S, Fukui N, Kawagoe H. Predictors of Place of Death for Japanese Patients with Advanced-Stage Malignant Disease in Home care Settings: A Nationwide Survey. Cancer 2004; 101(2): 421-9.

32. McLaughlin D, Hasson F, Kernohan WG, Waldron M, McLaughlin M, Cochrane B et al. Living and coping with Parkinson's disease: Perceptions of informal carers. J Palliat Med 2011; 25(2): 177-82.

33. Aabom B, Pfeiffer $P$. Why are some patients in treatment for advanced cancer reluctant to consult their GP? Scand J Prim Health 2009; 27(1): 58-62.

34. Jones RVH, Hansford J, Fiske J. Death from cancer at home: the carers' perspective. BMJ 1993; 306(6872): 249-51.

35. Campbell C, Nair Y, Maimane S, Sibiya Z. Supporting people with AIDS and their carers in rural South Africa: Possibilities and challenges. Health Place 2008; 14(3): 507-18.

36. Makoae MG. Food meanings in HIV and AIDS caregiving trajectories: Ritual, optimism and anguish among caregivers in Lesotho. Psychology, Health Medicine 2011; 16(2): 190-202.

37. Pallangyo E, Mayers P. Experiences of Informal Female Caregivers Providing Care for People Living With HIV in Dar es Salaam, Tanzania. J Assoc Nurses AIDS Care 2009; 20(6): 481-93.

38. Earnshaw S, Njongwe PZ, English B, Worku Z. The health and living conditions of children in child-headed households in siteki, Swaziland. East Afr J Public Health 2009; 6(1): 95-101.

39. Ingleton C, Payne S, Sargeant A, Seymour J. Barriers to achieving care at home at the end of life: transferring patients between care settings using patient transport services. J Palliat Med 2009; 23(8): 723-30. 
40. Figueiredo RM, Turato ER. Needs for assistance and emotional aspects of caregiving reported by aids patient caregivers in a da y-care unit in Brazil. Issues Ment Health Nurs 2001; 22(6): 633-43.

41. Ama NO, Seloilwe E. Estimating the cost of care giving on caregivers for people living with HIV and AIDS in Botswana: a cross-sectional study. J Int AIDS Soc 2010; 13(14): 1-8.

42. Moalosi G, Floyd K, Pharshwane J, Moeti T, Binkin N, Kenyon T. Costeffectiveness of home-based care versus hospital care for chronically ill tuberculosis patients, Francistown, Botswana. Suplemento Int J Tuberc lung Dis 2003; 7(9): 80-5.

43. Park C-H, Shin DW, Choi JY, Kang J, Baek YJI, Mo HN et al. Determinants of the burden and positivity of family caregivers of terminally ill cancer patients in Korea. Psycho-Oncology 2012; 21: 282-90.

44. Ryn M van, Sanders S, Kahn K, Houtven C van, Griffin JM, Martin M et al. Objective burden, resources, and other stressors among informal cancer caregivers: a hidden quality issue? Psycho-Oncology 2011; 20(1): 44-52.

45. Deshields TL, Rihane A, Potter P, Zhang Q, Kuhrik M, Kuhrik N et al. Psychosocial aspects of caregiving: perceptions of cancer patients and family caregivers. Support Care Cancer 2012; 20(2): 349-56.

46. Heide A van der, Vogel-Voogt E, Visser AP, Rijt CCD van der, Maas PJ van der. Dying at home or in an institution: perspectives of Dutch physicians and bereaved relatives. Support Care Cancer 2007; 15(12): 1413-21.

47. Milberg A, Strang P. Meaningfulness in palliative home care: An interview study of dying cancer patients' next of kin. Palliat Support Care 2003; 1(2): 171-80.

48. Uys LR. The practice of community caregivers in a home-based HIV/AIDS project in South Africa. J Clin Nurs Jan 2002; 11(1): 99-108

49. Schumacher KL, Stewart BJ, Archbold PG, Dodd MJ, Dibble SL. Family Caregiving Skill: Development of the Concept. Res Nurs Health 2000; 23(3): 191203.

50. Vallerand AH, Saunders MM, Anthony M. Perceptions of Control Over Pain by Patients with Cancer and Their Caregivers. Pain Manag Nurs 2007; 8(2): 55-63.

51. Bucher JA, Trostle GB, Moore M. Family Reports of Cancer Pain, Pain Relief, and Prescription Access. Cancer Pract 1999; 7(2): 71-8.

52. Oliver DP, Wittenberg-Lyles E, Demiris G, Washington K, Porock D, Day M. Barriers to Pain Management: Caregiver Perceptions and Pain Talk by Hospice Interdisciplinary Teams. Journal of Pain and Symptom Management 2008; 36(4): 37482.

53. Chepngeno-Langat G, Madise N, Evandrou M, Falkingham J. Gender differentials on the health consequences of care-giving to people with AIDS-related illness among older informal carers in two slums in Nairobi, Kenya. AIDS Care 2011; 23(12): 158694.

54. Foster G, Makufa C. Supporting children in need through a community-based orphan visiting programme. AIDS Care 1996; 8(4): 389-403.

55. Kuo C, Operario D, Cluver L. Global Public Health: An International Journal for Research, Policy and Practice. Global Public Health 2012; 7(3): 253-69.

56. Cameron JI, Franche R-L, Cheung AM, Stewart DE. Lifestyle Interference and Emotional Distress in Family Caregivers of Advanced Cancer Patients. Cancer 2002; 94(2): 521-7.

57. Motswasele LK. The experiences of informal care givers in home-based care in the ODI sub-district area in the North West Provice. Curationis 2008; 31(4): 24-30.

58. Jepson C, McCorkle R, Adler D, Nuamah I, Lusk E. Effects of home care on caregivers' psychosocial status. Image J Nurs Sch 1999; 31 (2): 2115-120. 
59. Shugarman LR, Buttar A, Fries BE, Moore T, Blaum CS. Caregiver Attitudes and Hospitalization Risk in Michigan Residents Receiving Home- and Community-Based Care. JAGS 2002; 50(6): 1079-85.

60 . Gysels M, Higginson IJ. Caring for a person in advanced illness and suffering from breathlessness at home: Threats and resources. Palliat Support Care 2009; 1(2): 15362.

61. Sit JWH, Wong TKS, Clinton M, LI LSW, Fong Y. Stroke care in the home: the impact of social support on the general health of family caregivers. J Clin Nurs 2004; 13(7): 816-24.

62. Fenga M, Feng J-Y, Chen T-C, Lu P-L, Ko N-Y, Chen Y-H. Stress, needs, and quality of life of family members caring for adults living with HIV/AIDS in Taiwan. AIDS Care 2009; 21(4): 482-9.

63. Grov EK, Fossa SD, Sørebø Ø, Dahl A. Primary caregivers of cancer patients in the palliative phase: A path analysis of variables influencing their burden. Soc Sci Med 2006; 63(9): 2429-39.

64. Lai H-L, Li Y-M, Lee L-H. Effects of music intervention with nursing presence and recorded music on psycho-physiological indices of cancer patient caregivers. J Clin Nurs 2012; 21(5-6): 745-56.

65. Tang W-R. Hospice family caregivers' quality of life. J Clin Nurs 2009; 18(18): 2563-72.

66. Tsigaroppoulos T, Mazaris E, Chatzidarellis E, Skolarikos A, Varkarakis I, Deliveliotis $\mathrm{C}$. Problems faced by relatives caring for cancer patients at home. Int $\mathrm{J}$ Nurs Pract 2009; 15(1): 1-6.

67. Hudson P, Quinn K, Kristjanson L, Thomas T, Braithwaite M, Fischer $J$ et al. Evaluation of a psycho-educational group programmed for family caregivers in homebased palliative care. Palliat Med 2008; 22(3): 270-80.

68. Hudson Peter, Thomas T, Quinn K, Cockayne M, Braithwaite M. Teaching Family Carers About Home-Based Palliative Care: Final Results from a Group Education Program. J Pain Symptom Manage 2009; 38(2): 299-308.

69. Gelesson DD, Hiraishi LY, Ribeiro LA, Pereira RS, Gutiérrez MGR, Domenico EBL. Significado da neutropenia e necessidades de cuidado em domicílio para os cuidadores de crianças com câncer. Rev Latino-am Enferm 2009; 17(6): 7 telas.

70. Grov EK, Eklund ML. Reactions of primary caregivers of frail older people and people with cancer in the palliative phase living at home. J Adv Nurs 2008; 63(6): 57685.

71. Ferrario SR, Cardillo V, Vicario F, Balzarini E, Zotti AM. Advanced cancer at home: caregiving and bereavement. Palliat Med 2004; 18(2): 129-36.

72. Comaru NRC, Monteiro ARM. O cuidado domiciliar à criança em quimioterapia na perspectiva do cuidador familiar. Rev Gaúcha Enferm 2008; 3(29): 423-30.

73. Siegel K, Raveis VH, Houts P, Mor V. Caregiver Burden and Unmet Patient Need. Cancer 1991; 68(5): 1131-40.

74. Milberg A, Strang P, Jakobsson M. Next of kin's experience of powerlessness and helplessness in palliative home care. Support Care Cancer 2004; 12(2): 120-8.

75. Kealey P, Mcintyre IG. An evaluation of the domiciliary occupational therapy service in palliative cancer care in a community trust: a patient and carers perspective. Eur J Cancer Care 2005; 14 (3): 232-43.

76. Keene J, Hope T, Fairburn CG, Jacoby R, Gedling K, Ware CJG. Natural history of aggressive behaviour in dementia. International journal of geriatric psychiatry. Int. J. Geriat. Psychiatry 1999; 14(7): 541-8.

77. Turato ER. Tratado de metodologia da pesquisa clínico-qualitativa: construção teórico-epistemológica, discussão comparada e aplicação nas áreas da saúde e humanas. $3^{\underline{a}}$ ed. Petrópolis, RJ: Vozes 2008. 
78. Foucault M. A arqueologia do saber. 7. ed. Rio de Janeiro: Forense Universitária 2004.

Recibido: 15 de julio de 2014; Aceptado18 de agosto 2014 